Collection SFN 11 (2010) 127-136

(C) Owned by the authors, published by EDP Sciences, 2010

DOI: $10.1051 / \mathrm{sfn} / 201011008$

\title{
Apports des techniques de diffusion de rayonnement à l'étude de la structure et de la dynamique des fluides magnétiques
}

\author{
E. Dubois
}

Laboratoire Physicochimie des Électrolytes, Colloïdes et Sciences Analytiques (PECSA), UPMC, UMR CNRS-UPMC-ESPCI 7195, 4 place Jussieu, case 51, 75005 Paris, France

\begin{abstract}
Résumé. Les liquides magnétiques, dispersions de nanoaimants dans des solvants, sont des dispersions colloïdales dans lesquelles le rôle des interactions dipolaires et l'influence d'un champ magnétique appliqué ont puêtre compris grâce à l'association (i) des techniques de diffusion de rayonnement, aussi bien statique que dynamique, (ii) d'autres techniques complémentaires du point de vue des échelles spatiales et temporelles et (iii) de systèmes modèles bien définis permettant d'identifier les paramètres clefs gouvernant les propriétés de ces dispersions. L'intérêt de cette association d'approches est illustré par plusieurs exemples de résultats.
\end{abstract}

\section{INTRODUCTION}

Les fluides magnétiques sont des suspensions colloïdales de nanoparticules magnétiques, en général d'oxydes, d'un diamètre de l'ordre de $10 \mathrm{~nm}$, dont les applications sont nombreuses et variées. Les liquides purs servent par exemple de joints tournants, l'une des premières applications, mais également comme agent de contraste en IRM, tandis qu'associés à un autre système, ils peuvent lui conférer des fontionnalités nouvelles. C'est le cas de telles particules ajoutées dans des colles, qui peuvent prendre grâce à un chauffage induit par les particules à distance par application de champs alternatifs appropriés [3,11, 17]. Les propriétés magnétiques des particules ajoutent une spécificité au système car chaque particule est un nanoaimant et des interactions magnétiques existent entre elles, de telle sorte que ces solutions colloïdales sont aussi des fluides dipolaires. Beaucoup étudiés dans la littérature pour leurs propriétés, ces liquides ont fait l'objet de peu d'études sur leur structure microscopique, qui est pourtant essentielle à comprendre et à contrôler pour maîtriser leurs propriétés. Etant données les échelles spatiales et temporelles concernées, la diffusion de rayonnement est un outil puissant et bien adapté pour comprendre la structure et la dynamique de ces milieux aux échelles des dimensions des nanoobjets et de la portée des interactions entre les objets, en relation avec les propriétés macroscopiques. Cependant, nous montrerons qu'il est nécessaire d'associer cette approche à d'autres techniques complémentaires, en illustrant par des travaux du laboratoire PECSA et de ses collaborateurs sur les liquides magnétiques synthétisés au laboratoire PECSA.

Dans les liquides purs se posent essentiellement les questions de l'influence du moment dipolaire sur les propriétés, en comparaison d'une suspension colloïdale classique, et de l'influence du champ magnétique, aussi bien sur la microstructure que sur la stabilité. Dans les systèmes mixtes, mélanges de particules et d'autres composés (polymères, tensio-actifs ...), se pose la question de leurs compatibilités. Dans tous les cas il est nécessaire de comprendre quels sont les paramètres clefs qui gouvernent leur comportement, ce qui nécessite l'utilisation de systèmes modèles bien définis.

This is an Open Access article distributed under the terms of the Creative Commons Attribution-Noncommercial License 3.0, which permits unrestricted use, distribution, and reproduction in any noncommercial medium, provided the original work is properly cited. 


\section{SYSTÈMES ET TECHNIQUES D'ÉTUDES}

\subsection{Systèmes}

Les liquides magnétiques sont des dispersions de nanoaimants permanents, le plus souvent d'oxyde de fer (magnétite $\mathrm{Fe}_{3} \mathrm{O}_{4}$ ou maghémite $\left.\gamma-\mathrm{Fe}_{2} \mathrm{O}_{3}\right)$ mais également d'oxydes mixtes $\left(\mathrm{MFe}_{2} \mathrm{O}_{4}\right.$ ) ou plus rarement de métaux $(\mathrm{Fe}, \mathrm{Co})$, dans un solvant polaire ou non. Les interactions attractives de van der Waals entre les particules ainsi que les interactions dipolaires magnétiques (en moyenne attractives) sont contrebalancées par des répulsions électrostatiques (solvants polaires uniquement) ou par des répulsions stériques. Dans tous les cas, les interactions dipolaires peuvent être quantifiées efficacement par le paramètre d'interaction dipolaire $\gamma$, qui compare l'énergie dipolaire à kT pour deux dipôles alignés :

$$
\gamma=\frac{\mu_{\circ} \mu^{2}}{k_{\mathrm{B}} T \bar{r}^{3}}=\mu_{\circ} \mu^{2} \frac{\pi d^{3}}{6} \frac{\Phi}{k T}
$$

où $\bar{r}$ est la distance interparticules moyenne. Après division par la fraction volumique $\Phi$, on obtient $\gamma / \phi$, qui caractérise les nanoparticules et leur comportement comme nous le verrons par la suite. Cette grandeur est déterminée expérimentalement par une mesure de susceptibilité $\chi=\chi_{o}$ à bas champ magnétique et interactions négligeables entre particules car $\gamma=3 \chi_{o}$ dans ces conditions [8]. La valeur expérimentale correspond alors à une moyenne sur toutes les tailles de particules, dont la polydispersité varie de 15 à $40 \%$.

Parmi ces systèmes, nous nous focaliserons ici sur des particules de maghémite $\left(\gamma-\mathrm{Fe}_{2} \mathrm{O}_{3}\right.$ dont l'intérêt est la stabilité chimique dans le temps et la simplicité, et dont nous maîtrisons la synthèse chimique et la dispersion. Nous avons essentiellement étudié deux systèmes modèles: (i) des particules recouvertes d'acide oléique dispersées dans le cyclohexane (répulsions stériques); (ii) des particules en milieu aqueux recouvertes de citrate, petite molécule qui assure une charge négative à $\mathrm{pH} 7$ (2 charges $/ \mathrm{nm}^{2}$, répulsions électrostatiques), avec des contreions sodium et une force ionique contrôlée par le citrate de sodium libre [Cit $t_{\text {libre }}$ [6]. Les résultats porteront ici sur ce deuxième cas. Dans ces systèmes, les diamètres des particules sont compris entre 4 et $12 \mathrm{~nm}$, la polydispersité varie de 15 à $40 \%$, [Cit ${ }_{\text {libre }}$ ] entre $0.003 \mathrm{M}$ et $0.1-0.3 \mathrm{M}$, et $6<\gamma / \phi<60$, ce qui permet de mimer les caractéristiques rencontrées dans les fluides standards commerciaux.

Comme tout système colloïdal, ils peuvent être considérés comme un analogue de système moléculaire à une échelle plus grande, de telle sorte que le diagramme usuel P-V devient un diagramme $\Pi-\Phi$ ou la $\Phi$ est l'analogue de $1 / \mathrm{V}$ et où la pression osmotique $\Pi$ est l'analogue de la pression P. Les caractéristiques du système, en particulier les interactions entre objets, sont intégrées dans le paramètre $\Pi$ donc dans ce diagramme.

\subsection{Méthodes expérimentales}

Si le diagramme $\Pi-\Phi$ est l'un des axes de contrôle des liquides, il faut cependant lui ajouter le champ magnétique $\mathrm{H}$, ce qui implique d'utiliser des techniques qui permettent de travailler sous champ et à différentes échelles spatiales. La Figure 1 résume l'ensemble des techniques et échelles accessibles dans le cas présent. Ainsi, du point de vue de la structure, la diffusion de rayonnement, bien adaptée aux échelles nanométriques, peut être complétée par la microscopie optique aux échelles microniques. Les techniques dynamiques aux échelles nanométriques, basées sur le rayonnement (ici X-Ray Photocorrelation Spectroscopy (XPCS) ou Neutron Spin Echo (NSE)) sont limitées en termes de gammes de dynamiques, qui sont de plus disjointes dans notre cas, mais la diffusion de lumière standard (LPCS) n'est pas utilisable en raison de la très forte absorption des particules dans le visible. Cependant, la technique de diffusion Rayleigh Forcée (FRS), basée sur la mesure de relaxation d'un gradient de concentration induit par un gradient de température (effet Soret), grâce à la diffraction du réseau de concentration induit, donne accès à une dynamique de translation [2]. Enfin, la biréfringence 


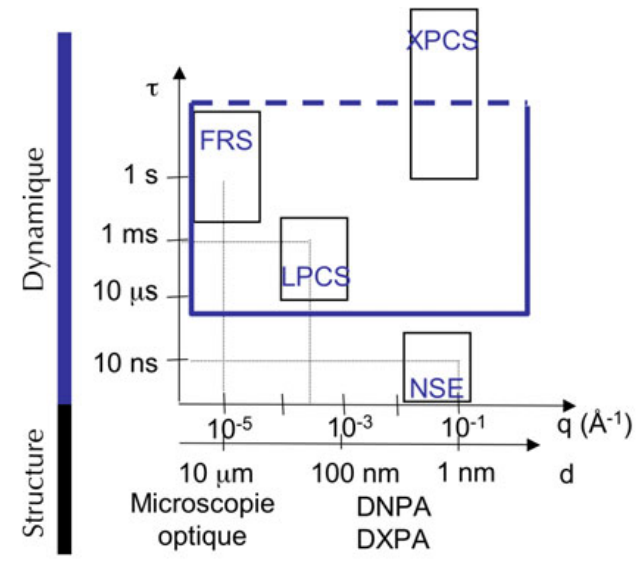

\begin{tabular}{|c|c|c|}
\hline \multicolumn{2}{|c|}{$\begin{array}{l}\text { Champ } \\
\text { magnétique H }\end{array}$} & $\begin{array}{l}\mathrm{mm} \\
\text { Échelle } \\
\text { spatiale }\end{array}$ \\
\hline Structure & $\begin{array}{l}\text { DNPA } \\
\text { DXPA } \\
\end{array}$ & $\begin{array}{c}\text { Microscopie } \\
\text { optique } \\
\end{array}$ \\
\hline $\begin{array}{l}\text { Dynamique } \\
\text { de translation }\end{array}$ & $\begin{array}{c}\text { Echo de spin de } \\
\text { neutrons, } \\
\text { Corrélation de } \\
\text { photons X (XPCS) }\end{array}$ & $\begin{array}{c}\text { Diffusion } \\
\text { Rayleigh Forcée }\end{array}$ \\
\hline $\begin{array}{l}\text { Dynamique } \\
\text { de rotation }\end{array}$ & \multicolumn{2}{|c|}{ Biréfringence magnéto optique } \\
\hline
\end{tabular}

Figure 1. Schéma des échelles spatiales et temporelles sondées dans ce travail dans les dispersions colloïdales magnétiques et des techniques associées. Dynamique de translation: les gammes spatiales et temporelles accessibles sont indiquées par les rectangles sur le schéma de gauche (trait fin): FRS (Forced Rayleigh Scattering), Light photocorrelation spectroscopy (LPCS), X-Ray Photocorrelation Spectroscopy (XPCS), Neutron Spin Echo (NSE). Dynamique de rotation: indiquée par le rectangle bleu (trait large), mesurée grâce aux propriétés magnéto-optiques des particules pour des temps supérieurs à la microseconde. Structure: techniques de diffusion de rayonnement aux petits angles (DNPA pour les neutrons et DXPA pour les rayons X).

magnéto-optique, qui utilise la biréfrigence de la solution induite sous champ magnétique (due à l'orientation des nanoparticules individuellement biréfringentes) permet de mesurer la dynamique de rotation des particules : on mesure la relaxation de la biréfringence à la coupure du champ grâce à la mesure d'une intensité transmise dans le visible [10]. L'intérêt de toutes ces techniques est la possibilité de les utiliser sous champ magnétique (ce qui n'est cependant pas évident pour la NSE). On peut alors obtenir des informations sur la structure et la dynamique de translation hors champ et sous champ.

\subsection{Neutrons ou rayons $X$ ?}

Les neutrons et les rayons $\mathrm{X}$ peuvent être en principe utilisés, cependant, interagissant différemment avec la matière, ils ne livrent pas toujours les mêmes informations sur le système étudié. En effet, les rayons X sont fortement absorbés par les particules d'oxydes de fer dans les énergies usuellement utilisées (le seuil d'absorption du fer est à $7.1 \mathrm{keV}$ ) ce qui peut induire un échauffement et une modification du système. En revanche, le contraste entre l'oxyde de fer et tous les solvants est bon. Au contraire, les neutrons ne sont pas absorbés, mais ils sont sensibles au moment magnétique des particules en plus de leur sensibilité aux noyaux atomiques. Ainsi l'intensité diffusée est la somme d'une contribution magnétique $\mathrm{I}_{\mathrm{M}}$ et d'une contribution nucléaire $\mathrm{I}_{\mathrm{N}}$ dont la séparation nécessite l'utilisation de neutrons polarisés ou l'association de neutrons et de rayons X. Cependant une différence importante existe entre les deux contributions: $\mathrm{I}_{\mathrm{N}}$ dépend de la nature du solvant, à savoir hydrogéné ou deutéré, tandis que $\mathrm{I}_{\mathrm{M}}$ n'en dépend pas. On peut alors montrer que le signal dans $\mathrm{H}_{2} \mathrm{O}$ est majoritairement nucléaire alors que le signal dans $\mathrm{D}_{2} \mathrm{O}$ est surtout magnétique (notons qu'on ne peut pas éteindre le signal nucléaire de ces particules dans un mélange de solvants par variation de contraste) [1, 8]. La détermination du signal magnétique permet d'obtenir des informations sur la taille magnétique et les interactions magnétiques, qui apparaissent attractives dans un système globalement répulsif, comme attendu [8]. L'analyse du signal nucléaire fournit des informations similaires à celles données par l'intensité diffusée par les rayons X. 


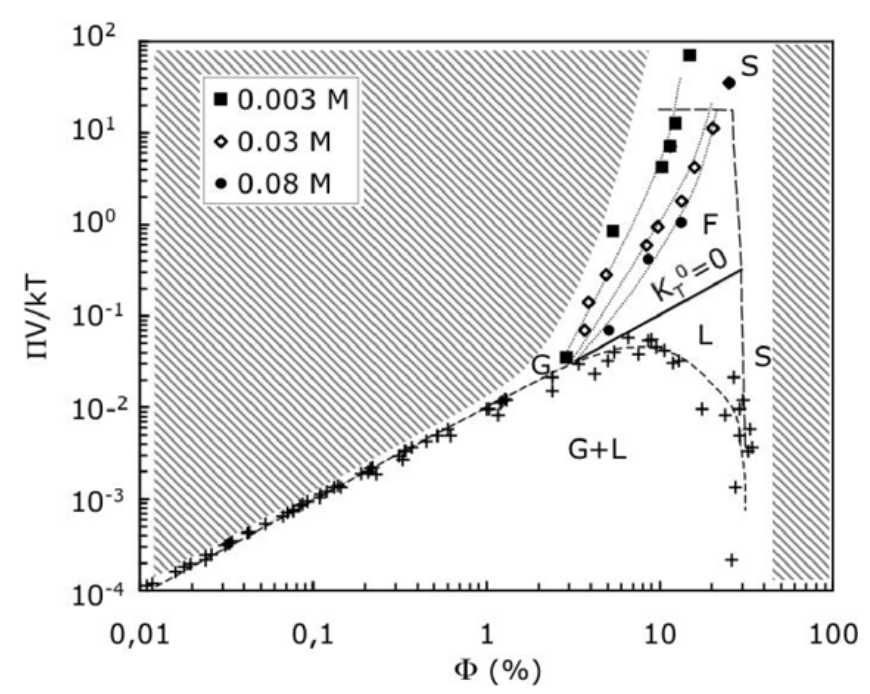

Figure 2. Diagramme de phase des dispersions. G: gaz, L: liquide, F: fluide, S: solide, zones hachurées: inaccessibles, croix: points expérimentaux de la courbe de coexistence gaz-liquide. Lignes pointillées: guides indiquant cette courbe de coexistence gaz-liquide et la limite entre les fluides et les solides. Trait plein: second coefficient du viriel $\mathrm{K}_{\mathrm{T}}^{\circ}$ nul. Les points expérimentaux reportés correspondent à une série de dispersions de particules d'un diamètre de $10 \mathrm{~nm}(\gamma / \phi=34)$ et chaque symbole correspond à une force ionique différente, indiquée dans la légende ([Cit $\left.]_{\text {libre }}\right)$.

Dans les travaux présentés ici, nous avons utilisé essentiellement la diffusion de neutrons en raison de l'absorption des rayons X. Cependant, pour les échantillons les plus concentrés, nous montrerons que le signal magnétique peut devenir gênant ce qui impose alors l'utilisation de rayons $\mathrm{X}$.

\section{LIQUIDES MAGNÉTIQUES PURS}

Couplée à la préparation de systèmes bien définis, l'ensemble des analyses par les techniques de diffusion et d'autres techniques complémentaires présentées plus haut ont permis d'aboutir au diagramme de phase $\Pi-\Phi$ montré sur la Figure 2. Nous allons illustrer la démarche sur certaines parties de ce diagramme, à savoir dans la zone du point critique, dans la zone fluide avec et sans champ magnétique, ainsi que dans la zone vitreuse.

\subsection{Zone critique}

La zone critique correspond au sommet de la courbe de coexistence entre le gaz et le liquide et le point critique donne la pression au dessus de laquelle on peut passer continđment du gaz au liquide sans séparation de phase, comme dans un système atomique. Notons que ce système est l'un des rares systèmes colloïdaux de nanoparticules solides stabilisées par des répulsions électrostatiques qui montre de telles transitions de type gaz-liquide. Cette particularité provient de la forme du potentiel, modifié par les interactions dipolaires magnétiques, dont la longue portée permet de stabiliser la phase liquide [5].

Cette zone autour du point critique a pu être étudiée grâce à la combinaison de DNPA et de microscopie optique qui associent deux échelles spatiales complémentaires [4]. Dans les deux cas, un échantillon situé dans la zone monophasique mais proche de la ligne de coexistence a été refroidi afin de passer la transition de façon finement contrôlable (on a une "équivalence" T-force ionique car $\Delta \mathrm{T} \simeq 10^{\circ} \mathrm{C}$ correspond à $\Delta\left[\mathrm{Cit}_{\text {libre }}\right] \simeq 0.01 \mathrm{~mol} / \mathrm{L}$ ). Les observations microscopiques montrent des échantillons clairement biphasiques loin de cette zone critique (une phase concentrée et une phase diluée 
en particules) tandis que de forts gradients de densité fluctuants dans le temps existent dans une plage de fractions volumiques $\Phi$ entre 6 et $12 \%$ et sur environ $10 \mathrm{~K}$ en température.

Dans les mêmes conditions, les mesures de facteur de structure par DNPA montrent que le potentiel entre objets dans cette zone est en moyenne faiblement attractif et les mesures de la dynamique de rotation permettent de conclure qu'il n'y a pas formation d'agrégats. Le potentiel d'interactions entre particules est donc de type sphères dures adhésives. La compressibilité obtenue par DNPA permet de déterminer une longueur de corrélation $\xi$ des fluctuations, dont les valeurs les plus grandes sont obtenues pour $\Phi=9 \%$, ce qui est cohérent avec la microscopie optique. Les mesures ne permettent cependant pas une détermination expérimentale fiable de l'exposant critique et une comparaison aux modèles car le point critique n'est pas assez bien défini du fait de la zone critique très large, ce qui peut avoir pour cause la polydispersité résiduelle des nanoparticules.

\subsection{Structure et dynamique des fluides sans champ magnétique}

La zone fluide correspond à la zone des hautes pressions osmotiques, située au dessus de la courbe de coexistence gaz-liquide, dans laquelle les échantillons sont monophasiques. La connaissance du comportement des dispersions dans cette zone s'est révélée essentielle pour comprendre leur comportement sous champ (cf section 3.3). Hors champ, ces échantillons sont monophasiques au microscope optique et la DNPA permet de montrer qu'ils ont une structure de type liquide répulsif : le facteur de structure montre une faible compressibilité et un pic qui correspond à la distance moyenne entre les particules, en accord avec la fraction volumique des dispersions. A basse fraction volumique $\Phi$, la compressibilité $\chi_{T}$, déterminée à partir de la valeur du facteur de structure extrapolé à $\mathrm{q}=0$, permet de déterminer le second coefficient du Viriel $K_{T}^{o}$ à partir de $\chi_{T}=1 /\left(1+K_{T}^{o} \Phi\right)$. D'autre part la mesure du coefficient de diffusion de translation $D_{t r}$ en fonction de $\Phi$ par la technique FRS permet de déterminer également ce même coefficient $K_{T}^{o}$ car $D_{t r}(\Phi)=D_{o}\left(1+\left(K_{T}^{o}-K_{f}\right) \Phi\right)$. $D_{o}$ est mesuré par DLS dans un système très dilué et on suppose pour $K_{f}$, terme hydrodynamique, la valeur des sphères dures. Les valeurs de $K_{T}^{o}$ obtenues à partir de la statique et de la dynamique sont en bon accord et correspondent à un système répulsif [13]. Par ailleurs, l'étude de la dynamique de rotation des particules ne montre pas d'évolution significative de $\tau_{\text {rot }}$ dans la gamme des basses $\Phi$, ce qui signifie que les particules restent sous forme de particules isolées dans toute cette zone du diagramme de phase. En champ nul, les particules sont donc dispersées de façon individuelle, et interagissent par des répulsions sans formation de chaŤnes de particules.

\subsection{Structure et dynamique des fluides sous champ magnétique}

Lorsqu'un champ magnétique constant et homogène est appliqué à ces dispersions colloïdales de particules magnétiques, il y a à l'échelle des particules orientation des moments magnétiques dans la direction du champ lorsque le champ augmente (paramagnétisme géant de la solution). Du point de vue macroscopique, il peut se produire une séparation de phase entre une phase diluée et une phase concentrée en raison de l'augmentation des attractions en moyenne sous champ, qui conduit à franchir la ligne de coexistence gaz-liquide si l'échantillon se trouve assez près de cette ligne dans le diagramme de phase $[9,12]$. Nous nous plaçons ici dans le cas de dispersions sous champ qui restent au dessus de cette ligne, afin d'étudier la microstructure sous champ dans la zone monophasique.

L'analyse par DNPA sous champ montre des spectres plus ou moins anisotropes selon les situations (Fig. 3(a) et (b)), qui sont analysés en extrayant les intensités dans les directions parallèles et perpendiculaires au champ. L'anisotropie de l'intensité diffusée peut provenir en principe (i) du signal diffusé magnétique anisotrope sous champ, (ii) du facteur de forme des particules qui pourraient présenter une anisotropie (iii) du facteur de structure $S$ (q) donc de l'organisation des particules. Le signal magnétique étant négligeable, et le facteur de forme expérimental ne présentant pas d'anisotropie, on en déduit que l'anisotropie provient du facteur de structure S(q). Ce dernier montre que la compressibilité 

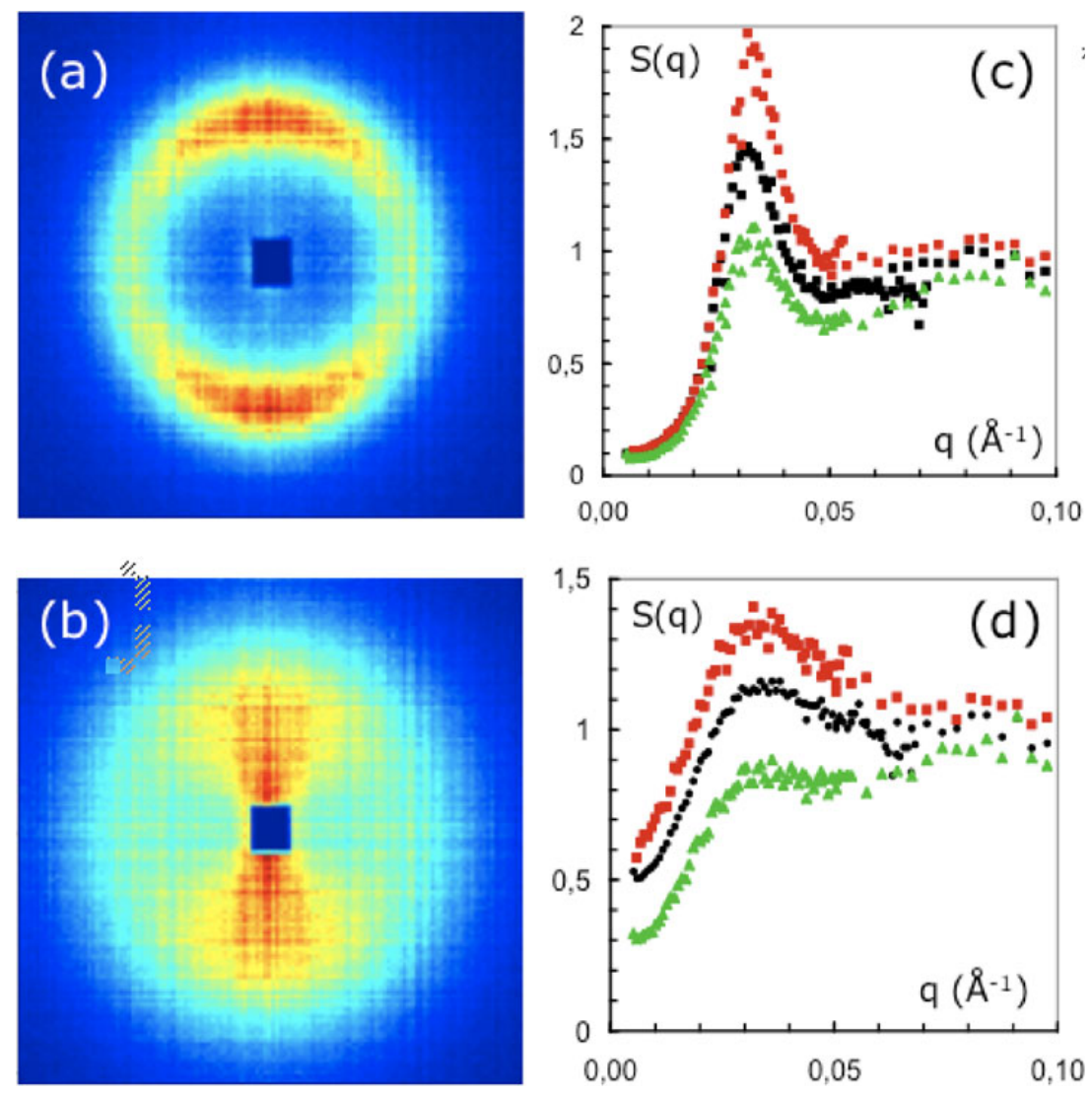

Figure 3. Structure sous champ: (a) et (b): Intensité brute diffusée sur le détecteur; (c) et (d): facteurs de structure déterminés expérimentalement hors champ (carrés noirs), parallèlement au champ (triangles verts) et perpendiculairement au champ (carrés rouges). (a) et (c): basse force ionique/haute pression osmotique; (b) et (d): haute force ionique/basse pression osmotique.

est anisotrope ainsi que l'amplitude du pic de corrélation, correspondant à la distance moyenne entre particules, qui reste isotrope avec la précision de la DNPA (Fig. 3(c) et (d)).

A petits q, le comportement peut être expliqué par un modèle de type champ moyen qui montre que la compressibilité est modifiée par un terme isotrope et attractif et par un terme anisotrope et répulsif qui est maximal parallèlement au champ et nul perpendiculairement au champ. Ce deuxième terme, lié aux fluctuations anisotropes du champ macroscopique, est dominant, et induit des interactions globalement plus répulsives dans la direction parallèle au champ, ce qui est contre-intuitif. Par ailleurs, ces termes dépendent de $\mathrm{H}, \Phi$ et $\gamma / \Phi$. Ainsi l'anisotropie à champ fort des spectres dépend de $(\gamma / \Phi) / K_{o}^{T}$, donc du rapport de l'interaction dipolaire par rapport à l'interaction totale en champ nul, dominée dans la zone étudiée par les répulsions électrostatiques. Cela signifie donc que la structure est contrôlée par la taille des particules qui modifie fortement les interactions dipolaires, ainsi que par la position de l'échantillon dans le diagramme de phase, essentielle à maŤtriser pour contrôler la structure des suspensions et leurs propriétés (Fig. 3(c) et (d)).

A l'échelle locale du pic de $\mathrm{S}(\mathrm{q})$, la solution est plus structurée dans la direction perpendiculaire à $\mathrm{H}$ et on ne voit aucune trace de la formation de chaînes de particules. Ce dernier point est confirmé par la simulation par dynamique brownienne d'un système modèle représentatif du système expérimental $[14,16]$, qui montre la même anisotropie de $S(q)$ et l'absence de chaŤnes, qui peuvent 
cependant se former dans des systèmes constitués de particules de plus fort moment magnétique, non représentatif de ces systèmes expérimentaux.

Dans ces conditions, la dynamique sous champ peut être étudiée à l'échelle microscopique par FRS (q plus petits que la DNPA) et à l'échelle locale de la distance entre objets par NSE (autour du pic du facteur de structure). Les phénomènes dynamiques apparaissent très fortement dépendants de l'échelle spatiale sondée: la FRS montre une forte anisotropie du coefficient de diffusion de translation qui peut être expliquée par le même modèle que la compressibilité: $D_{t r}^{/ /}>D_{o}>D_{t r}^{\perp}$. En revanche, à l'échelle locale, la NSE ne présente pas d'anisotropie et les simulations confirment qu'elle est faible [15]. Ces résultats montrent que l'anisotropie observée résulte de phénomènes collectifs.

L'ensemble de l'étude permet donc de mettre en évidence l'absence de chaŤnes de particules dans ces systèmes sous champ, qui présentent une structure anisotrope dont la dynamique dépend de l'échelle spatiale.

\subsection{Transition vitreuse}

Lorsque la concentration en particules augmente, les dispersions deviennent solides, que les interactions soient attractives ou répulsives (Fig. 2). Nous avons exploré l'approche de cette transition dans le cas des interactions répulsives entre particules. A haute fraction volumique, une transition fluide/solide réversible conduit à un solide amorphe dont la structure reste celle d'un liquide, comme le montrent la DNPA ou la DXPA. Il s'agit donc d'une transition vitreuse, le cristal n'étant pas formé en raison de la polydispersité des particules. Du fait de la bonne connaissance des interactions et du diagramme de phase de ces dispersions, elles constituent de bons modèles pour l'étude de la transition vitreuse. En effet, elles permettent de modifier le système à travers des paramètres non accessibles dans les systèmes utilisés dans l'étude de la transition vitreuse, à savoir le potentiel d'interaction entre particules, et surtout le champ magnétique extérieur, paramètre qui permet de modifier le système à distance, en particulier d'introduire un potentiel d'interaction anisotrope.

Dans ces systèmes, la DNPA doit être remplacée par la DXPA en raison de la pollution dans certains cas par le signal magnétique. La NSE ne permet pas de mesurer la dynamique des particules qui devient trop lente. En revanche, si le système devient suffisamment lent (dynamique $>$ à $10 \mathrm{~s}$ typiquement dans notre cas), sa dynamique peut être mesurée par l'XPCS, et on peut utiliser les mesures optiques pour déterminer la dynamique de rotation (cf Fig. 1). On montre alors qu'il y a un blocage dynamique de la rotation des objets par blocage des sphères effectives lorsque $\Phi$ augmente et que la dynamique de translation devient également lente. L'XPCS permet alors d'étudier le vieillissement de cette dynamique dans le temps ainsi que l'influence d'un champ magnétique. Sous champ, la dynamique devient anisotrope dans ces dispersions vitreuses, alors qu'elle restait isotrope dans le cas des dispersions fluides [18].

\subsection{Bilan}

L'étude des dispersions de particules magnétiques par les techniques de diffusion associées à d'autres type de mesures sur des systèmes bien définis a donc permis de montrer que les paramètres clefs qui contrôlent leurs propriétés sont la taille des particules, qui contrôle $\gamma / \Phi$, la pression osmotique $\Pi$ et la fraction volumique $\Phi$. Sans champ magnétique, le comportement des dispersions est proche de celui d'un système non magnétique, cependant la balance des interactions conduit à l'existence de transitions de type gaz-liquide. En revanche, sous champ magnétique, on induit soit une séparation de phase, ce qui modifie beaucoup les propriétés des dispersions, soit une anisotropie de structure dans un système monophasique sans formation de chaînes. La diversité des observations possibles provient de la localisation de l'échantillon dans le plan $\Pi-\Phi$, ce qui est contrôlé essentiellement par les interactions entre les particules. 


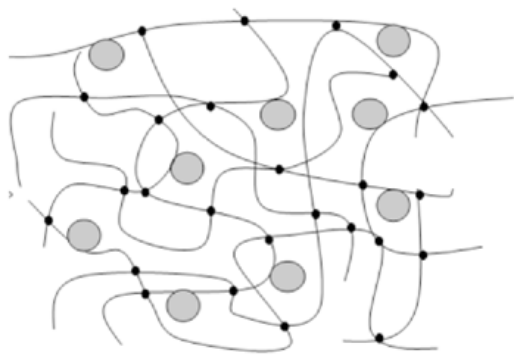

Cas A

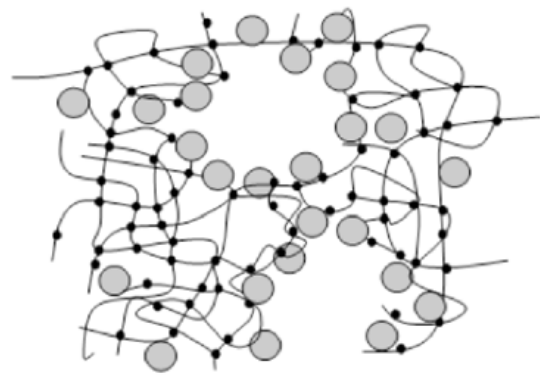

Cas B

Figure 4. Microstructure des ferrogels dans deux cas : $\mathrm{A}=R_{\Pi}<1$ et $R_{s}<1 ; \mathrm{B}=R_{s} \simeq 1$ et $R_{\Pi}>0.2$.

L'utilisation d'un système modèle a permis de comprendre ce qui gouverne le comportement de telles dispersions et les conclusions obtenues peuvent être transposées à d'autres systèmes tels que ceux fabriqués à partir de particules composées d'autres ferrites magnétiques (ferrite de cobalt, de cuivre...) ou à des systèmes dans des solvants non polaires dans lesquels la stabilisation est stérique et non électrostatique.

\section{APPLICATION À UN SYSTÈME MIXTE : EXEMPLE DES FERROGELS}

L'étude du cas des ferrogels illustre l'intérêt de connaître la structure des particules au sein d'un système mixte, dont elles constituent l'un des composants, et l'apport de la diffusion de rayonnement, là encore couplée à d'autres techniques, qui se complètent parfaitement [7].

Ces systèmes sont constitués de particules magnétiques dans un gel de polymère réticulé, ce qui permet de combiner les propriétés des deux systèmes et d'envisager la modification des propriétés mécaniques des polymères. Composés d'acrylamide réticulé par de la N,N'-methylenebisacrylamide, qui polymérise dans le milieu contenant les particules magnétiques nanométriques, les gels sont immergés après synthèse dans un bain jusqu'à ce que l'équilibre soit atteint, phase pendant laquelle le gel peut gonfler et relarguer des particules selon les cas.

Dans ce cas, la DNPA des particules dans le système où le signal du polymère a été éteint dans un mélange $\mathrm{H}_{2} \mathrm{O} / \mathrm{D}_{2} \mathrm{O}$ approprié donne une image de la structure du système, que l'on peut associer à l'étude de la dynamique de rotation des particules dans le gel. On peut alors montrer que les paramètres clefs sont $R_{\Pi}=\Pi_{\text {ferrofluide }} / \Pi_{\text {hydrogel }}$ et $R_{s}=d / \xi$, où l'hydrogel est le gel sans particules, d le diamètre des particules et $\xi$ la taille des mailles du réseau de polymère. $P i_{\text {ferrofluide }}$ est la pression osmotique du ferrofluide pur et $P i_{\text {hydrogel }}$ est la pression osmotique de l'hydrogel pur avant gonflement.

Si $R_{\Pi}<1$ et $R_{S}<1$ (cas A), la DNPA montre que le facteur de structure des particules dans le gel est proche de celui du ferrofluide pur de même concentration. Par ailleurs, les mesures de biréfringence magnéto-optique montrent que la plupart des particules sont bloquées en rotation et que le temps caractéristique de rotation de celles qui tournent est proche de celui des particules du fluide pur. On peut alors conclure à l'existence d'une structure homogène dans laquelle les particules sont adsorbées sur le polymère et restent dans le gel, conduisant alors à une modification contrôlée du module élastique (voir schéma sur la Fig. 4).

Si $R_{s} \simeq 1$ et $R_{\Pi}>0.2$ (cas $\mathrm{B}$ ), il y a un fort relargage des particules du gel lors du gonflement, ce qui conduit à une faible fraction volumique en particules dans le matériau final. Cependant, la DNPA montre un pic de structure ainsi que des hétérogénéités à petits q, donc à grandes distances. Par ailleurs la majorité des particules est bloquée en rotation. On en déduit alors l'existence dans ce cas d'une structure hétérogène dans laquelle les particules sont adsorbées sur la surface de poches liquides, dont toutes les particules libres ont été relarguées (voir schéma sur la Fig. 4). 
La DNPA couplée à l'analyse de la rotation des particules et à des mesures physo-chimiques permet donc de montrer que la structure finale de ces ferrogels et leurs propriétés sont contrôlées par les caractéristiques du ferrofluide utilisé comparées à celles de l'hydrogel, ce qui montre la nécessité de connaŤtre le diagramme de phase des ferrofluides et de savoir s'y positionner à volonté.

\section{CONCLUSIONS}

Les études présentées brièvement ici illustrent l'intérêt de la diffusion de rayonnement couplée à des systèmes modèles bien définis et à d'autres techniques pour étudier la structure et la dynamique de ces systèmes de nanoparticules magnétiques dispersées dans des solvants. Elles ont permis de mettre en évidence la très forte influence du diamètre $d$ des nanoparticules constituant les systèmes, en raison de la rapide variation des interactions dipolaires avec $d$. On a montré que les ferrofluides classiques dont les particules ont un diamètre compris entre 4 et $12 \mathrm{~nm}$ ne forment pas d'agrégats ou de chaŤnes de particules hors champ et sous champ, mais présentent une structure et une dynamique anisotrope selon les échelles. Au final, les propriétés du système sont gouvernées par la localisation dans un diagramme $\Pi-\Phi$ qui constitue un diagramme de phase nécessaire à connaŤtre pour contrôler le système. Lorsque la position d'un échantillon dans ce plan n'est pas connue ou maŤtrisée, comme par exemple dans les produits commerciaux, une grande variabilité des mesures et propriétés peut être observée. Ainsi la localisation des échantillons dans ce diagramme est un prérequis nécessaire pour la formulation de liquides magnétiques purs ou intégrés dans des systèmes complexes.

\section{Remerciements}

Ces résultats sont le fruit du travail de nombreux chercheurs: - des étudiants en thèse : Guillaume Mériguet, Elie Wandersman, Alberto Galicia; - des chercheurs du laboratoire: Régine Perzynski, Gilles Demouchy, Vincent Dupuis, Marie Jardat, Valérie Cabuil; - des chercheurs des différents grands instruments dans lesquels ont été faites les expériences: François Boué (LLB), Fabrice Cousin (LLB), Aymeric Robert (ESRF, SLAC), Bela Farago (ILL), Mikhael Avdeev (Dubna), Yuriy Chuschkin (ESRF).

\section{Références}

[1] M.V. Avdeev, E. Dubois, G. Mériguet, E. Wandersman, V.M. Garamus, A.V. Feoktystov, and R. Perzynski. Small-angle neutron scattering analysis of a water-based magnetic fluid with charge stabilization: contrast variation and scattering of polarized neutrons. Journal of Applied Crystallography, 42:1009-1019, 2009.

[2] J.C. Bacri, A. Cebers, A. Bourdon, G. Demouchy, B.M. Heegard, B. Kashevsky, and R. Perzynski. Transient grating in a ferrofluid under magnetic field: effect of magnetic interaction on the diffusion coefficient of translation. Phys. Rev. E, 52(4):3936, 1995.

[3] B. Berkowski, editor. Magnetic fluids and application handbook. Begell House (UNESCO), 1996.

[4] F. Cousin, E. Dubois, and V. Cabuil. Approach of the critical point of gas-liquid transitions in an electrostatically stabilized colloidal suspension. J. Chem. Phys., 115:6051-6057, 2001.

[5] F. Cousin, E. Dubois, and V. Cabuil. Tuning the interactions of a magnetic colloidal suspension. Phys. Rev. E, 68:021405-1 to 021405-9, 2003.

[6] E. Dubois, V. Cabuil, F. Boué, and R. Perzynski. Structural analogy between aqueous and oily magnetic fluids. J. Chem. Phys., 111(15):7147-7160, 1999.

[7] A. Galicia, F. Cousin, E. Dubois, O. Sandre, V. Cabuil, and R. Perzynski. Static and dynamic structural probing of swollen polyacrylamide ferrogels. Soft Matter, 5:2614-2624, 2009.

[8] F. Gazeau, F. Boué, E. Dubois, and R. Perzynski. Static and quasi-elastic neutron scattering on biocompatible ionic ferrofluids: Magnetic and hydrodynamic interactions. J. Phys.: Condens. Matter, 15:S1305-1334, 2003. 
[9] F. Gazeau, E. Dubois, J.C. Bacri, F. Boué, A. Cebers, and R. Perzynski. Anisotropy of the structure factor of magnetic fluids under a field probed by small angle neutron scattering. Phys. Rev. E, 65:031403-1 to $15,2002$.

[10] E. Hasmonay, E. Dubois, J.C. Bacri, R. Perzynski, and V.I. Stepanov Yu.L. Raikher. Static magneto-optical birefringence of size-sorted $\gamma-f e_{2} O_{3}$ nanoparticle. Eur. Phys. J. B, 5:859-867, 1998.

[11] H. Lohse. Rapid bonding of composite parts : Automotive. JEC composites, 19:41-44, 2005.

[12] G. Mériguet, F. Cousin, E. Dubois, F. Boué, A. Cebers, B. Farago, and R. Perzynski. What tunes the structural anisotropy of magnetic fluids under a magnetic field? J. Phys. Chem. B, 110(43784386), 2006.

[13] G. Mériguet, E. Dubois, A. Bourdon, G. Demouchy, V. Dupuis, and R. Perzynski. Forced rayleigh scattering experiments in concentrated magnetic fluids: Effect of interparticle interactions on the diffusion coefficient. J. Magn. Magn. Mat., 289:39-42, 2005.

[14] G. Mériguet, E. Dubois, M. Jardat, A. Bourdon, G. Demouchy, V. Dupuis, B. Farago, R. Perzynski, and P. Turq. Understanding the structure and the dynamics of magnetic fluids: coupling of experiment and simulation. J. Phys.: Condens. Matter, 18:S2685-S2696, 2006.

[15] G. Mériguet, M. Jardat, E. Dubois, B. Farago, and R. Perzynski. Diffusion in magnetic fluids under an external field: neutron spin echo experiments and brownian dynamics simulations. soumis.

[16] G. Mériguet, M. Jardat, and P. Turq. Structural properties of charge-stabilized ferrofluids under a magnetic field: A brownian dynamics study. J. Chem. Phys., 121:6078-6085, 2004.

[17] S. Odenbach, editor. Magnetically controllable fluids and their applications. Springer Verlag (Berlin), 2003.

[18] A. Robert, E. Wandersman, E. Dubois, V. Dupuis, and R. Perzynski. Glassy dynamics and aging in a dense ferrofluid. Europhys. Lett., 75(5):764-770, 2006. 